\title{
An Interpolating Model Predictive Control Strategy with Application to a Waste Treatment Plant
}

\author{
S. Joe Qin* and Víctor M. Martínez \\ Department of Chemical Engineering \\ The University of Texas at Austin \\ Austin, TX 78712, USA \\ Fax: 512-471-7060 \\ Email: qin@che.utexas.edu
}

\author{
Bjarne A. Foss \\ Department of Engineering Cybernetics \\ The University of Trondheim - NTH \\ Norway \\ Email: baf@itk.unit.no
}

\begin{abstract}
In this paper a new model predictive control (MPC) strategy, applicable to a set of nonlinear systems, is proposed and the use of it is demonstrated on a model of a waste treatment reactor. The MPC strategy is an extension of earlier work in optimization-based control [2]. The motivation for the study is to search for approaches to nonlinear MPC without having to solve the full nonlinear problem. We restrict our problem by defining a nonlinear model set as a convex combination of a set of bounding linear models. The weighting factors between the models can be a function of the states and/or inputs. At a given time-instant we compute an optimal future control sequence for each of the bounding linear models. A novel feature is that all models must obey the constraints for each of the control sequences. The reason for these additional constraints is that they provide us with feasibility guarantees. It also is a means of robustifying the MPC. The final control sequence is found by interpolating the control sequences derived from the optimization problems. There are different possible approaches for choosing the interpolation variables. Provided the optimization criterion and the constraint sets for the control variables and states are convex, the proposed control algorithm involves only convex optimization problems. The interpolating MPC strategy is applied to a waste treatment reactor, where the process dynamics are nonlinear and time-varying depending on the disturbance. Linearization is carried out to obtain bounding models for the process. The interpolating MPC is designed based on the bounding models. Through the example we demonstrate significant improvements over a standard quadratic MPC strategy based on linear models.
\end{abstract}

\section{Introduction}

The combined use of dynamic models and optimization for process control offers a concept in which process knowledge can be linked to operational goals formulated by some optimization criterion. This concept has seen widespread use, particularly through the applications of model predictive control (MPC). MPC refers to a class of algorithms where an optimization problem is solved repetitively, at every new time-instant. Only the first part of the computed control sequence is applied to the system since a new optimal control sequence is computed and applied at the next timestep. Several reviews of MPC technology exist, see for example Lee [4], Rawlings et al. [8] and Qin and Badgwell [7], the latter emphasizing industrial use of the technology. The interaction between control and optimization is discussed in an illuminating way in Mayne [6].

*Author to whom all correspondence should be addressed.
A popular MPC strategy is based on linear dynamic models and linear constraints on the control inputs and system outputs. Nonlinear optimizing control has been studied by Rawlings et al. [8] and Genceli and Nikalaou [3]. Further, some approaches were described by Bequette [1] in a somewhat earlier paper. These control strategies normally result in a non-convex optimization problem.

In this paper we explore an MPC strategy based on nonlinear models. In particular, the goal is to derive an approach which can offer a smooth transition from linear MPC to nonlinear MPC. By smooth transition we mean an approach which can deal with nonlinear processes but does not invoke non-convex optimization, which may be difficult to implement. This is particularly important from an industrial viewpoint as it simplifies the transition from the application of linear to nonlinear MPC. The smooth transition is accomplished by constraining the nonlinear optimization problem along three axes. First, the set of nonlinear 
models is limited to a convex, nonlinear interpolation of linear models. Second, constraints are added to the optimization problem to enhance feasibility of an optimal solution for a set of bounding linear models. Third, the control sequence is computed as a convex combination of control sequences based on the bounding linear models.

The remainder of this paper is structured as follows: In the next section we formulate the problem. The theoretical foundation, model development, and control design for the proposed control algorithm are then developed. In section 4 a waste treatment example is used to test the effectiveness of the proposed method. Finally, conclusions are given at the end of the paper.

\section{Problem Formulation}

In this section we formulate the problem of MPC with multiple linear models. We define a set of linear statespace models:

$$
\begin{aligned}
\Sigma_{j}: \quad \mathbf{h}_{j}(\mathbf{x}(i+1), \mathbf{x}(i), \mathbf{u}(i)) & = \\
\tilde{\mathbf{x}}(i+1)-\mathbf{A}_{j} \tilde{\mathbf{x}}(i)-\mathbf{B}_{j} \tilde{\mathbf{u}}(i) & =0 \\
j \in I_{J}=\{1,2, \cdots, J\} &
\end{aligned}
$$

where $\tilde{\mathbf{x}}(i)=\mathbf{x}(i)-\overline{\mathbf{x}}_{j}$ and $\tilde{\mathbf{u}}(i)=\mathbf{u}(i)-\overline{\mathbf{u}}_{j}$ are deviation variables. Since the ultimate goal is to deal with nonlinear processes, the actual variables $\{\mathbf{x}(i), \mathbf{u}(i)\}$ are used rather than the deviation variables. The linear models in $\Sigma_{j}$ necessarily have different steady states $\left\{\overline{\mathbf{x}}_{j}, \overline{\mathbf{u}}_{j}\right\}$ to calculate the deviation. The model inputs and state variables are constrained as follows,

$$
\begin{aligned}
& \mathbf{u}(i) \in U \subseteq \Re^{m_{u}} \\
& \mathbf{x}(i) \in X \subseteq \Re^{m_{x}}
\end{aligned}
$$

where $U$ and $X$ are convex sets. We assume the set of nonlinear processes are interpolation of the $J$ linear models,

$$
\begin{aligned}
\Sigma_{w}= & \left\{\mathbf{h}_{w}: \mathbf{h}_{w}(\mathbf{x}(i+1), \mathbf{x}(i), \mathbf{u}(i))=\right. \\
& \Sigma_{j=1}^{J} w_{j}(\mathbf{x}(i), \mathbf{u}(i)) \mathbf{h}_{j}(\mathbf{x}(i+1), \mathbf{x}(i), \mathbf{u}(i))= \\
& \left.0 \quad \forall j \in I_{J}, \quad w_{j} \in W\right\} \\
W= & \left\{w_{j} \in \mathcal{C}: w_{j}(\mathbf{x}(i), \mathbf{u}(i)) \in[0,1], \Sigma_{j} w_{j}==1\right. \\
& \forall \mathbf{x}(i) \in X \forall \mathbf{u}(i) \in U\}
\end{aligned}
$$

where $\mathbf{h}_{w}$ is a nonlinear function constructed as convex combinations of $\mathbf{h}_{j}, j \in I_{J}$. It should be noted that the continuous function $w_{j}$ in general will depend on the states and the control inputs. Provided that some limitations are placed on the nonlinear process model, such a model can be approximated arbitrarily well by increasing $J[5]$.
The model predictive control objective function is defined as follows,

$$
\phi(\pi, \chi)=\sum_{i \in I_{N}} l(\mathbf{x}(i+1), \mathbf{u}(i))
$$

where

$$
\begin{aligned}
\pi= & \{\mathbf{u}(0), \ldots, \mathbf{u}(N-1)\} \subseteq \Pi=U \times \ldots \times U \\
\chi= & \{\mathbf{x}(1), \ldots, \mathbf{x}(N)\} \subseteq \mathcal{X}=X \times \ldots \times X \\
l: \Re^{m_{u} \times m_{y}} \rightarrow \Re^{+} \text {is a convex function } & \\
& i \in I_{N}=\{0, \ldots, N-1\}
\end{aligned}
$$

The optimality criterion is defined on a time horizon from 0 to $N$. The control input $\mathbf{u}(i)$ is constant during the time span $[i, i+1)$. Since $U$ and $X$ are convex sets, $\Pi$ and $\mathcal{X}$ are convex sets. Further, $\phi$ is convex since $l$ is convex. The criterion function (5) does not cover all possible criteria; penalizing changes in the control input is for example not included. This type of change does not influence the results in this paper as long as the criterion function remains convex.

Assume that the current state variables are available, i.e.,

$$
\mathbf{x}(0) \text { - given }
$$

The problem we want to address is to minimize (5) with respect to $\pi$ based on the different constraints discussed above, hence we want to solve

$$
\pi^{\circ}=\arg \min _{\pi \in \Pi} \phi(\pi, \chi)
$$

subject to the constraints $\chi \in \mathcal{X}$ and (6) using one of the models $\left(\Sigma_{j}, j \in I_{J}\right.$ or $\left.\Sigma_{w}\right)$. The control sequence is typically parameterized as follows,

$$
\pi=\{\mathbf{u}(0), \ldots, \mathbf{u}(M), \ldots, \mathbf{u}(M)\}, M \leq N-1
$$

This means that the control input is constant during the last part of the control sequence. The minimization problem ( 7 ) is solved repetitively, at each timestep, with new initial conditions. Only the first control value of the $\pi$ sequence is actually applied to the process.

A nonlinear MPC formulation would minimize the criterion (5) subject to Eq. (4). Since Eq. (4) is nonlinear, the resulting optimization is in general nonconvex and a globally optimal solution is difficult to find in real-time control. A typical linear MPC formulation is to minimize the criterion (5) subject to one of the linear models in $\Sigma_{j}, j \in I_{J}$. The linear MPC solution, while easily solvable, is valid only in the small vicinity of the steady state around which the linear model is derived. 


\section{Interpolating Model Predictive Control}

\subsection{Feasibility of the Interpolation}

We propose an interpolating model predictive control (IMPC) which solves for $J$ linear MPC problems and the final control sequence is calculated via interpolation. First of all, we solve for $J$ control sequences

$$
\pi_{j}^{o}=\arg \min _{\pi \in \Pi} \phi\left(\pi, \chi_{j}\right)
$$

subject to

1. $\chi \in \mathcal{X}$ for all linear models in (1); and

2. using the particular model $\left(\Sigma_{j}\right)$ as the internal model to calculate $\chi_{j}$.

The above item 1 is necessary to guarantee the actual nonlinear process is within the constraints. This is shown in Theorem 1.

The interpolating MPC control sequence is defined as follows,

$\Pi_{\alpha}=\left\{\pi_{\alpha}: \pi_{\alpha}=\Sigma_{j=1}^{J} \alpha_{j} \pi_{j}^{o} \forall \alpha_{j} \in[0,1], \Sigma_{j=1}^{J} \alpha_{j}=1\right\}$

The control sequence $\pi_{\alpha}$ forms the basis for the controller. It should be noted that $\pi_{\alpha}$ is feasible in input since it is based on interpolation within a convex set, i.e., $\Pi_{\alpha} \subseteq \Pi$. $\left\{\alpha_{j}, j \in I_{J}\right\}$ may in general vary from one time instant to another.

An important issue is whether the interpolated control sequence $\pi_{\alpha}$ will make the state variable sequence

$$
\begin{aligned}
\mathcal{X}_{w}^{\alpha}= & \left\{\chi_{w}^{\alpha}: \chi_{w}^{\alpha}=\left\{x_{w}^{\alpha}(1) \ldots, x_{w}^{\alpha}(N)\right\},\right. \\
& \left.\forall h_{w} \in \Sigma_{w}, \pi_{\alpha} \in \Pi\right\}
\end{aligned}
$$

feasible, i.e., $\mathcal{X}_{w}^{\alpha} \in \mathcal{X}$. $\mathcal{X}_{w}^{\alpha}$ denotes the set of the state variable sequence by applying $\pi_{\alpha}$ to the process $\Sigma_{w}$. Here we provide the following theorem to guarantee feasibility for $J$ models, but the proof is omitted for brevity. The special case of $J=2$ is shown in Foss and Qin (1996).

Theorem 1 Assuming $J$ control sequences $\pi_{j}^{o}$ which are solved from $\mathrm{Eq}$. (9), then $\mathcal{X}_{w}^{\alpha} \in \mathcal{X}$ if $\left\{\Sigma_{j}, j \in I_{J}\right\}$ are finite impulse response (FIR) models with $\left\{\mathbf{A}_{j}, \mathbf{B}_{j}, \mathbf{C}_{j}\right\}$ given as follows,

$$
\begin{aligned}
\mathbf{A}_{j} & =\left(\begin{array}{ccccc} 
& \tilde{\mathbf{I}} & & 0 \\
\mathbf{H}_{j 1} & \ldots & \mathbf{H}_{j L} & 0
\end{array}\right) \\
\tilde{\mathbf{I}} & =\left(\begin{array}{ccccc}
0 & \ldots & \ldots & 0 \\
\mathbf{I} & 0 & \ldots & 0 \\
\vdots & \ddots & & 0 \\
0 & \ldots & \mathbf{I} & 0
\end{array}\right)
\end{aligned}
$$

$$
\mathbf{B}_{j}=\left(\begin{array}{c}
\mathbf{I} \\
0 \\
\vdots \\
0 \\
\mathbf{H}_{j 0}
\end{array}\right)
$$

and $\mathbf{C}_{j}=(0, \ldots, 0, \mathbf{I})^{T} .\left\{\mathbf{H}_{j 0}, \mathbf{H}_{j 1}, \cdots, \mathbf{H}_{j L}\right\}$ are the FIR coefficient matrices.

The theorem is due to the formulation of the minimization problem (9) where the constraints for all linear bounding models are considered. The significance of the theorem is that the interpolated solution $\pi_{\alpha}$ is feasible no matter how $\left\{\alpha_{j}, j \in I_{J}\right\}$ is chosen. This is important in searching for optimal $\left\{\alpha_{j}\right\}$ where the problem becomes unconstrained. In the subsequent sections we will discuss how to obtain the bounding linear models, $\left\{w_{j}(\mathbf{x}(i), \mathbf{u}(i))\right\}$ and $\left\{\alpha_{j}\right\}$ to implement the IMPC algorithm.

\subsection{The Bounding Linear Models and $\left\{w_{j}, j \in I_{J}\right\}$}

There are in general two approaches to finding the bounding linear models (i) through first principles of the process and linearization, and (ii) through system identification. Both approaches derive models that are valid in a small vicinity of the steady states, which can be described by the state variables or throughput variables of the process. Assuming the first principles model is available in the following form,

$$
\begin{aligned}
\frac{d \mathbf{x}(t)}{d t} & =\mathbf{f}(\mathbf{x}(t), \mathbf{u}(t)) \\
0 & =\mathbf{g}(\mathbf{y}(t), \mathbf{x}(t), \mathbf{u}(t))
\end{aligned}
$$

where $\mathbf{f}$ is a continuous function of $\mathbf{x}$ and $\mathbf{u}$, linearization can be carried out around the steady states by specifying lower and upper bounds for each state variable $\left\{\bar{x}_{k}^{L}, \bar{x}_{k}^{H} ; k=1, \ldots, m_{x}\right\}$. Since the state variables uniquely determine the system, the parameterization for $\left\{w_{k}\right\}$ can be given as follows:

$$
w_{k}=w_{k}\left(x_{k}, \bar{x}_{k}^{L}, \bar{x}_{k}^{H}\right) \quad k=1, \ldots, m_{x}
$$

For example, a linear weighting function is:

$$
w_{k}=\frac{x_{k}-\bar{x}_{k}^{L}}{\bar{x}_{k}^{H}-\bar{x}_{k}^{L}} \quad \in[0,1]
$$

The interpolation is:

$$
y_{k}^{w}=\left(1-w_{k}\right) y_{k}^{L}+w_{k} y_{k}^{H}
$$

If the process state $x_{k} \rightarrow \bar{x}_{k}^{L}, w_{k} \rightarrow 0$ and the interpolation $y_{k}^{w} \rightarrow y_{k}^{L}$; if the process state $x_{k} \rightarrow \bar{x}_{k}^{H}$, 
$w_{k} \rightarrow 1$ and the interpolation $y_{k}^{w} \rightarrow y_{k}^{H}$. Note that the interpolation has to be done based on FIR models to satisfy Theorem 1 . The FIR models can be converted from the state space models. With this interpolation strategy, the maximum number of models are $2^{m_{x}}$ and the maximum number of weighting factors $w_{k}$ is $m_{x}$. In practice, not all states are actively varying in a wide region, which can be considered as $\bar{x}_{k}^{L}=\bar{x}_{k}^{H}$, significantly reducing the number of models and weighting factors. Further, varying along some states may not include nonlinear behavior. The former will be demonstrated with the waste treatment reactor later in this paper. If a state variable is not directly measurable, an observer can be designed.

If system identification is used to build the bounding linear models (1), a local or a global cost function can be used (Johansen and Foss, 1993). For each region of the throughput variables, a linear model is identified. The models weights are similar to those given in Eqs. (14), (15), and (16). One of such approaches is reported in Johansen and Foss (1993). The approach based on throughput variables normally requires a smaller number of models and the modeling effort only involves linear system identification.

\subsection{Finding the optimal $\left\{\alpha_{j}, j \in I_{J}\right\}$}

Although $\pi_{\alpha}$ is feasible for all $\pi_{\alpha} \in \Pi_{\alpha},\left\{\alpha_{j}\right\}$ can be chosen to optimize the control performance at each time step. One approach is to solve the following minimization problem:

$$
\pi_{\alpha}^{o}=\arg \min _{\left\{\alpha_{j}, j \in I_{J}\right\}} \phi\left(\pi_{\alpha}, \chi_{w}^{\alpha}\right)
$$

subject to

$$
\alpha_{j} \in[0,1] ; \quad \sum_{j=1}^{J} \alpha_{j}=1
$$

Although this optimization problem is not necessarily convex, the dimension of the search is typically low. For the case of two bounding models, which is expected to be typical in practice, the search is one-dimensional. Therefore, a global optimization over $\left\{\alpha_{j}\right\}$ can be implemented with little computational effort.

In the case that the above optimization is not tractable, a schedule between $\alpha_{j}$ and $w_{j}$ may be provided. One approach is to choose different values for $w_{j}$ and $\alpha_{j}$ and create an empirical relation between them, which would yield a minimum $\phi$. Another approach is to set up an analytical relation between $\alpha_{j}$ and $w_{j}$, i.e.,

$$
\alpha_{j}=\alpha_{j}\left(w_{j}\right)
$$

In general, if the process state $\mathbf{x} \rightarrow \overline{\mathbf{x}}_{j}^{L}, w_{j} \rightarrow 1$. In this case, we want $\pi_{\alpha} \rightarrow \pi_{j}^{L}$, which requires $\alpha_{j} \rightarrow 1$.
Similarly, if $w_{j} \rightarrow 0, \alpha_{j} \rightarrow 0$. Therefore, a monotonic relation is expected. As a particular case, we may specify $\alpha_{j}=w_{j}$. The case of a prescribed schedule between $w_{j}$ and $\alpha_{j}$ may be referred to as gain-scheduling $M P C$.

\section{Application to a Waste Treat- ment Reactor}

\subsection{Modeling}

In this section we use a waste treatment reactor to demonstrate the interpolating MPC approach. The reactor has waste water (alkali), acid, catalyst and oxidizer inflows. The objective is to control the $\mathrm{pH}$ at a given value so that the oxidation reaction can take place. The nonlinear model for the reactor is given by

$$
\begin{gathered}
\frac{d \xi}{d t}=\frac{1}{V}\left(v_{1} c_{1}-v_{i} \xi\right) \\
\frac{d \zeta}{d t}=\frac{1}{V}\left(v_{2} c_{2}-v_{i} \zeta\right) \\
{\left[H^{+}\right]^{4}+\left(\zeta+K_{1}\right)\left[H^{+}\right]^{3}+} \\
\left.\left(K_{1}\left(\zeta-0.5 \xi+K_{2}\right)-K_{w}\right)\right)\left[H^{+}\right]^{2}+ \\
\left(K_{1}\left[K_{2}(\zeta-\xi)-K_{w}\right]\right)\left[H^{+}\right]-K_{1} K_{2} K_{w}=0
\end{gathered}
$$

and

$$
\mathrm{pH}=-\log _{10}\left[H^{+}\right]
$$

where each variable is described in Table 1.

\begin{tabular}{|c|c|}
\hline Variable & Description \\
\hline$v_{1}$ & acid stream flow rate \\
$c_{1}$ & acid concentration in the acid stream \\
$\xi$ & concentration of the acid in the reactor \\
$v_{2}$ & alkali flow rate \\
$c_{2}$ & alkali concentration in the base stream \\
$\zeta$ & concentration of the base in the reactor \\
$t$ & time \\
$V$ & reactor volume \\
$v_{3}$ & oxidizer flow rate \\
$v_{4}$ & catalyst flow rate \\
$v_{i}$ & $v_{1}+v_{2}+v_{3}+v_{4}$ \\
{$\left[H^{+}\right]$} & concentration of hydrogen ion \\
$K_{w}$ & water equilibrium constant \\
$K_{1}$ & sulfuric acid first dissociation constant \\
$K_{2}$ & sulfuric acid second dissociation constant \\
\hline
\end{tabular}

Table 1: Model variables description for the waste treatment model 
Linearization of the above non-linear model yields the following state space model:

$$
\begin{gathered}
\dot{\mathrm{x}}=\mathrm{Ax}+\mathrm{Bu} \\
\mathbf{y}=\mathrm{Cx}
\end{gathered}
$$

where

$$
\begin{aligned}
\mathbf{x} & =\left[\begin{array}{c}
\tilde{\xi} \\
\tilde{\zeta}
\end{array}\right], \quad \mathbf{y}=\mathrm{pH}, \quad \mathbf{u}=\left[\begin{array}{c}
\tilde{v}_{1} \\
\tilde{v}_{2} \\
\tilde{c}_{1} \\
\tilde{c}_{2}
\end{array}\right], \\
\mathbf{A} & =\frac{1}{V}\left[\begin{array}{cc}
-\bar{v}_{i} & 0 \\
0 & -\bar{v}_{i}
\end{array}\right], \\
\mathbf{B} & =\frac{1}{V}\left[\begin{array}{cccc}
\left(\bar{c}_{1}-\bar{\xi}\right) & -\bar{\xi} & \bar{v}_{1} & 0 \\
-\bar{\zeta} & \left(\bar{c}_{2}-\bar{\zeta}\right) & 0 & \bar{v}_{2}
\end{array}\right], \\
\mathbf{C} & =\frac{1}{\ln (10) \frac{\partial f}{\partial\left[H^{+}\right]_{0}}}\left[\begin{array}{ccc}
\frac{1}{\left[\bar{H}^{+}\right]} \frac{\partial f}{\partial \zeta} & \frac{1}{\left[\bar{H}^{+}\right]} \frac{\partial f}{\partial \xi} 0
\end{array}\right]
\end{aligned}
$$

and

$$
\begin{aligned}
\frac{\partial f}{\partial\left[H^{+}\right]_{0}=} & 4\left[\bar{H}^{+}\right]^{3}+3\left(\bar{\zeta}+K_{1}\right)\left[\bar{H}^{+}\right]^{2}+ \\
& \left.2\left(K_{1}\left(\bar{\zeta}-0.5 \bar{\xi}+K_{2}\right)-K_{w}\right)\right)\left[\bar{H}^{+}\right]+ \\
& K_{1}\left(K_{2}(\bar{\zeta}-\bar{\xi})-K_{w}\right) \\
\frac{1}{\left[\bar{H}^{+}\right]} \frac{\partial f}{\partial \xi_{0}=} & -0.5 K_{1}\left[\bar{H}^{+}\right]-K_{1} K_{2} \\
\frac{1}{\left[\bar{H}^{+}\right]} \frac{\partial f}{\partial \zeta_{0}}= & {\left[\bar{H}^{+}\right]^{2}+K_{1}\left[\bar{H}^{+}\right]+K_{1} K_{2} }
\end{aligned}
$$

where variables with “ " represent deviation variables and those with " " represent steady states. Since there are two state variables, at most four bounding models are needed. Those are given in Table 2.

\begin{tabular}{|c|c|c|}
\hline$\zeta \backslash \xi$ & $w_{1}=0$ & $w_{1}=1$ \\
\hline$w_{2}=0$ & $\left\{\bar{\xi}^{L}, \bar{\zeta}^{L}\right\}$ & $\left\{\bar{\xi}^{H}, \bar{\zeta}^{L}\right\}$ \\
& $\left\{\mathbf{A}_{00}, \mathbf{B}_{00}, \mathbf{C}_{00}, \mathbf{x}_{00}\right\}$ & $\left\{\mathbf{A}_{10}, \mathbf{B}_{10}, \mathbf{C}_{10}, \mathbf{x}_{10}\right\}$ \\
$w_{2}=1$ & $\left\{\bar{\xi}^{L}, \bar{\zeta}^{H}\right\}$ & $\left\{\bar{\xi}^{H}, \bar{\zeta}^{H}\right\}$ \\
& $\left\{\mathbf{A}_{01}, \mathbf{B}_{01}, \mathbf{C}_{01}, \mathbf{x}_{01}\right\}$ & $\left\{\mathbf{A}_{11}, \mathbf{B}_{11}, \mathbf{C}_{11}, \mathbf{x}_{11}\right\}$ \\
\hline
\end{tabular}

Table 2: Model Bounds

The weighting factors are specified as follows:

$$
\begin{array}{ll}
w_{1}=\frac{\xi-\bar{\xi}^{L}}{\bar{\xi}^{H}-\bar{\xi}^{L}} ; \quad w_{1} \in[0,1] \\
w_{2}=\frac{\zeta-\bar{\zeta}^{L}}{\bar{\zeta}^{H}-\bar{\zeta}^{L}} ; \quad w_{2} \in[0,1]
\end{array}
$$

and the interpolation is given by:

$$
\mathbf{x}_{w}=\left(1-w_{2}\right)\left[\left(1-w_{1}\right) \mathbf{x}_{00}+w_{1} \mathbf{x}_{10}\right]+w_{2}\left[\left(1-w_{1}\right) \mathbf{x}_{01}+w_{1} \mathbf{x}_{11}\right]
$$

which is consistent with Table 2. The above equation is the interpolated nonlinear representation of the process, which is bounded by $\left[\mathbf{x}_{00}, \mathbf{x}_{10}\right] \times\left[\mathbf{x}_{01}, \mathbf{x}_{11}\right]$. Once the bounds on the state variables are determined, the corresponding input variables can be calculated as follows:

$$
\begin{aligned}
\bar{v}_{1} & =\frac{c_{2} \bar{\xi}}{c_{1} c_{2}-c_{2} \bar{\xi}-c_{1} \bar{\zeta}}\left(v_{3}+v_{4}\right) \\
\bar{v}_{2} & =\frac{c_{1} \bar{\zeta}}{c_{1} c_{2}-c_{2} \bar{\xi}-c_{1} \bar{\zeta}}\left(v_{3}+v_{4}\right)
\end{aligned}
$$

For this particular process, we choose the bounds as given in Table 3 . Since the range for $\left[\bar{\zeta}^{L}, \bar{\zeta}^{H}\right]=$ $[0.1340,0.1345]$ is small, we neglect the variation in $\zeta$ and consider the two models for $w_{2}=0$ in Table 3 only.

\begin{tabular}{|c|c|c|}
\hline$\zeta \backslash \xi$ & $w_{1}=0$ & $w_{1}=1$ \\
\hline \multirow{3}{*}{$w_{2}=0$} & {$\left[\bar{\xi}^{L}, \bar{\zeta}^{L}\right]=$} & {$\left[\bar{\xi}^{H}, \bar{\zeta}^{L}\right]=$} \\
& {$[0.1373,0.1340]$} & {$[0.1673,0.1340]$} \\
& {$\left[\bar{v}_{1}, \bar{v}_{2}\right]=$} & {$\left[\bar{v}_{1}, \bar{v}_{2}\right]=$} \\
& {$[0.9599,81.15]$} & {$[1.337,92.78]$} \\
& & \\
$w_{2}=1$ & {$\left[\bar{\xi}^{L}, \bar{\zeta}^{H}\right]=$} & {$\left[\bar{\xi}^{H}, \bar{\zeta}^{H}\right]=$} \\
& {$[0.1373,0.1345]$} & {$[0.1673,0.1345]$} \\
& {$\left[\bar{v}_{1}, \bar{v}_{2}\right]=$} & {$\left[\bar{v}_{1}, \bar{v}_{2}\right]=$} \\
& {$[1.192,101.18]$} & {$[[1.720,119.8]$} \\
& & \\
\hline
\end{tabular}

Table 3: Steady state values for the four bounding models

\subsection{Control Design and Results}

To design the IMPC controller for the reactor, we use $v_{1}$ as the manipulated variable, $v_{2}$ the measured disturbance, $v_{3}$ and $v_{4}$ unmeasured disturbances. For each of the two models in the first row of Table 3 , the following constraints are specified:

$$
\begin{aligned}
v_{1} & \in\left[\begin{array}{ll}
0 & 2
\end{array}\right] \\
v_{2} & \in\left[\begin{array}{ll}
80 & 95
\end{array}\right] \\
\xi & \in\left[\begin{array}{ll}
0.13 & 0.17
\end{array}\right]
\end{aligned}
$$

The control sequence based on the two bounding models, $\pi_{00}^{\circ}$ and $\pi_{10}^{o}$, are solved via quadratic programming. The control horizon for the manipulated vari- 
able is 10 and the sample rate is 8 minutes. The objective function is:

$$
\phi=\sum_{i=1}^{N} y^{2}(i)+v_{1}^{2}(i-1)
$$

where $N$ is chosen as 45 . The interpolation of the control sequence is given by the following schedule:

$$
\alpha_{1}=w_{1}=\frac{\xi-\bar{\xi}^{L}}{\xi^{H}-\xi^{L}}
$$

and

$$
\pi_{\alpha}^{o}=\left(1-\alpha_{1}\right) \pi_{00}^{o}+\alpha_{1} \pi_{10}^{o}
$$

We simulate the control responses for setpoint and disturbance changes. The $\mathrm{pH}$ setpoint is changed from 3.66 to 3.0 and the alkali flow changes from $81.15 \mathrm{l} / \mathrm{min}$ to $89.27 \mathrm{l} / \mathrm{min}$ as measured disturbance. The control responses for the $\mathrm{pH}$ and acid flow are shown in Figure 1.
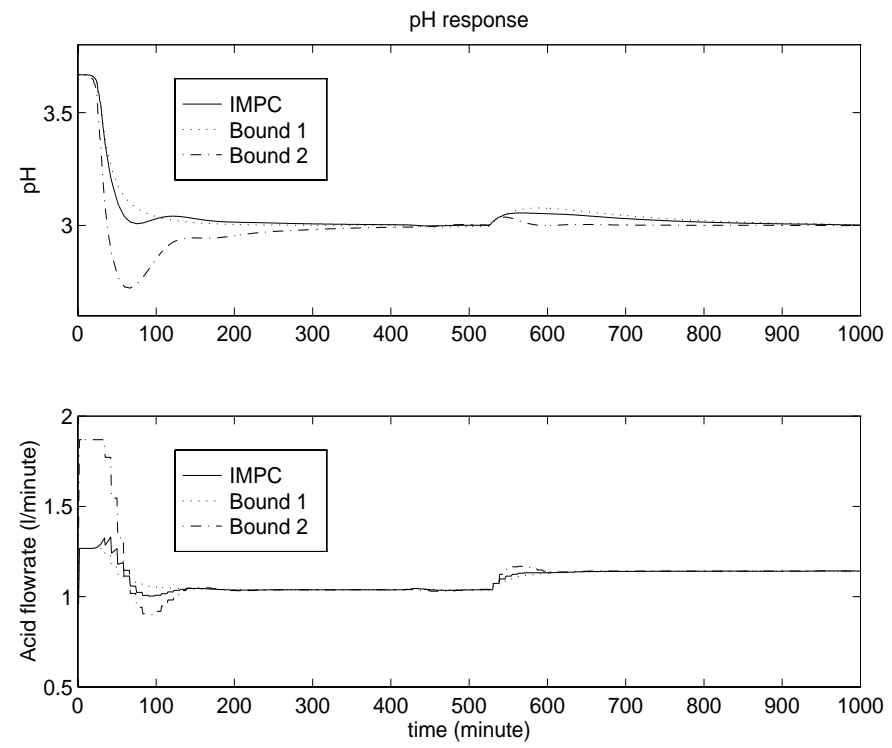

Figure 1: Control responses for $\mathrm{pH}$ and acid flow. "Bound 1" indicates the response for $\alpha=0$ and "Bound 2" for $\alpha_{1}=1$.

It is seen that the interpolating MPC performs consistently better in response to setpoint and disturbance changes. Importantly, the control variable response to the interpolated manipulated variable move is essentially bounded by the two linear control responses, regardless of the mismatch between the nonlinear process and the interpolated model.

\section{Conclusions}

A new interpolating MPC strategy is proposed for a set of nonlinear processes. The control move is based on a convex combination of control profiles generated from a set of linear bounding models.

Feasibility of the interpolated control is guaranteed in the strategy for FIR bounding models. The formulation of the problem avoids the non-convexity of a general nonlinear MPC. The on-line computation time is in the same order of magnitude as a linear MPC.

The waste treatment example demonstrates that the interpolating MPC is effective in handling rather nonlinear processes. Although the nonlinear process is not exactly a convex combination of bounding linear models, the interpolated MPC strategy demonstrates robust performance in the presence of setpoint and disturbance changes. Stability of the interpolating MPC is under study. Further work will extend the results to more general state space model representation.

\section{References}

[1] B. W. Bequette. Nonlinear control of chemical processes: A review. Ind. Eng. Chem. Res., 30:1391$1413,1991$.

[2] B. Foss and S. J. Qin. Interpolating optimizing process control. Journal of Process Control, 7, 1997.

[3] H. Genceli and M. Nikalaou. Design of robust constrained model-predictive controllers with volterra series. AIChE Journal, 41:2098-2107, 1995.

[4] J.H.Lee. Recent advances in model predictive control and other related areas. In Preprints $C P C-V$, Lake Tahoe, USA, 1996.

[5] T. A. Johansen and B. A. Foss. Constructing narmax models using armax models. Int. J. Control, 58:1125-1153, 1993.

[6] D. Q. Mayne. Optimization in model based control. In Preprints IFAC Symposium DYCORD, Helsingor, Denmark, 1995.

[7] S. Joe Qin and T.A. Badgwell. An overview of industrial model predictive control technology. In Preprints CPC-V, Lake Tahoe, USA, 1996.

[8] J. B. Rawlings, E. S. Meadows, and K. R. Muske. Nonlinear model predictive control: A tutorial and survey. In Preprints IFAC Symposium ADCHEM, Kyoto, Japan, pages 203-214, 1994. 